\title{
Pitch angle control of wind turbine systems in cold weather conditions using $\mu$ robust controller
}

\author{
Tahere Pourseif $^{1} \cdot$ Ali Afzalian ${ }^{1}$
}

Received: 24 September 2016/ Accepted: 8 March 2017/Published online: 20 March 2017

(c) The Author(s) 2017. This article is an open access publication

\begin{abstract}
Wind turbine is a complex nonlinear system that the exact model of this system is not available, so it can be represented as an uncertain system. Thus, the control of this system is an important topic. Most of methods that design controllers for wind turbines consider these systems in suitable weather condition. While many turbines work in cold weather condition, in this paper, wind turbine is considered in cold weather, and in ice on turbine blades are considered as model uncertainties. A robust controller is designed for the wind turbine, in order to control the pitch angle. Performance of the proposed controller is evaluated in a comparison study.
\end{abstract}

Keywords Wind turbine - Ice formation on wind turbine blades · PID controller - Robust control - Robust $\mu$ control . Reduced order controller

\section{Nomenclature}

$P_{\mathrm{e}} \quad$ Electrical power of generator $(\mathrm{KW})$

$\omega_{\mathrm{r}} \quad$ Angular speed of rotor $(\mathrm{rad} / \mathrm{s})$

$J_{\mathrm{r}} \quad$ Rotor inertia $(\mathrm{kg})$

$T_{\mathrm{r}} \quad$ Rotor torque (N M)

$\omega_{\mathrm{g}} \quad$ Angular speed of generator $(\mathrm{rad} / \mathrm{s})$

$J_{\mathrm{g}} \quad$ Generator inertia $\left(\mathrm{kg} \mathrm{m}^{2}\right)$

$T_{\mathrm{g}} \quad$ Generator torque (N M)

$D_{\mathrm{r}} \quad$ Damping coefficient of drive train $\left(\mathrm{s}^{-1}\right)$

$K_{\mathrm{r}} \quad$ Spring coefficient of drive train $(\mathrm{N} / \mathrm{M})$

Tahere Pourseif

t.pourseif@gmail.com

Ali Afzalian

Afzalian@sbu.ac.ir

1 Dept. of Electrical Engineering, Abbaspour School of Eng., Shahid Beheshti University, Tehran, Iran
$N_{\mathrm{g}} \quad$ Gear ration (-)

$\tau_{\beta} \quad$ Pitch actuator time constant (s)

$\tau_{\mathrm{T}} \quad$ Generator time constant (s)

$f \quad$ Frequency $(\mathrm{Hz})$

$v \quad$ Wind speed $(\mathrm{m} / \mathrm{s})$

$\rho \quad$ Air density $\left(\mathrm{kg} \mathrm{m}^{3}\right)$

\section{Introduction}

Increase in world population leads to enhanced consumption of electrical energy. Decrease in fossil fuels and environmental pollution encourages researchers to find new ways to generate electrical energy $[1,2]$.

Renewable energy is delivered from natural processes that are replenished constantly and are unpredictable. Therefore, it is difficult to control. This energy is applicable because of its being inexpensive, easy availability, and purity of the energy. Wind turbines are used to convert the kinetic energy of wind into electrical energy. Different control approaches are proposed to adjust the wind turbine speed for efficient power generation and to keep the turbine components within designed speed and torque limits $[1,3]$.

Considering turbine performance and its different operation areas, this paper aimed at designing controllers in the third operation area [4]. To be more precise, various types of control strategies are introduced to control and maximize power in this area. In [5], a fuzzy controller is designed to convert wind into optimal electrical energy. Demonstrating the stability of the system is demanding in this method due to the nonlinearity of the process. Therefore, an adaptive fuzzy controller is proposed in [6]. In that method, the adaptation process is accomplished through fuzzy rules and the stability of the system is guaranteed by choosing a suitable Lyapunov function [6]. In [7], a robust 
controller is designed which utilizes the T-S fuzzy model to stabilize the nonlinear wind turbine system. A drawback inherent in this method is the difficulty in determining the appropriate Lyapunov function to prove the stability This method suffers from the same flaw as the adaptive fuzzy controller. A PID controller as the most widely used control scheme in industry is proposed in [8]. Some advantages can be enumerated for this method, including its simple structure and easy implementation. In this method, if the system works in a steady-state operating point, the pitch angle will be controlled. It should be noted that nonlinear and complex dynamics of the system are not appraised since the system works in the operating point. This bespeaks that the linearization of the nonlinear model of wind turbine makes us ignore the small deviations around the operating point, noise, and the disturbance of the process. In [9], a sliding mode controller as a robust controller is used to control power and to adjust the speed of the rotor and the wind turbine generator. Due to the nonlinear characteristics of the controller, only two uncertainties including spring constant and damping coefficient have been considered and applied to the wind turbine system. The main drawback of this method is the chattering phenomenon in the sliding surface. In [10], a robust controller is designed to consider all of the uncertainties. These uncertainties include the uncertainty resulting from the linearization process and small deviations, and uncertainties from spring constant and damping coefficient. These methods are used to control the pitch angle and maximize electrical power generation [11].

In many instances, the performance of the wind turbine is scrutinized in suitable weather conditions. However, a robust controller is designed in this article to maintain the performance of the wind turbine in bad weather conditions, such as snow or when rain droplets freeze on the turbine blades [12]. In these circumstances, as the turbine blades freeze, the rotor mass changes and the electric power reduces. Therefore, the controller is designed to adjust the speed of the generator after applying the uncertainties in the inertia and other factors $[13,14]$. In light of the pitch angle and the rotor speed, the type of wind turbine used in this paper has variable rotor speed and variable pitch angle. The adjustment of the variable pitch angle and the variable rotor speed, respectively, results in the maximization of the electrical power and minimization of the turbine's dynamic load. It is note worthy that the adjustment of the variable rotor speed not only minimizes the turbine's dynamic load, but also increases the system's lifetime $[15,16]$. The objective of designing the controller is to maximize the electrical power production at low wind speed and to maintain it at high wind speed. Compared to other publications on wind turbine, in this paper, for the first time, the uncertainty that arises from icing of wind blade is considered, and then robust controller is designed.

The paper is organized as follows: Section 2 articulates the modeling of the wind turbine system and related equations. The impact of cold weather on the performance of wind turbine is explained in Sect. 3 that aims to study and evaluate the uncertainties of the system and based on the conclusions made in this section, robust $\mu$ and reduced order $\mu$ control systems are designed in Sect. 4 to control the blade angle. In Sect. 5, the simulation results of designed robust controller are compared with a PID control system, and finally conclusions made in Sect. 6.

\section{Wind turbine model}

This section introduces the wind model and the wind turbine model.

\section{Wind model}

Effective wind speed is a nonlinear stochastic process that is approximated by a linear model to simplify and satisfy the control objectives [11]. There are two terms in the wind model:

$V=V_{\mathrm{t}}+V_{\mathrm{m}}$

The turbulent term $V_{\mathrm{t}}$ can be modeled by

$$
\left(\frac{\dot{V}_{t}}{\ddot{V}_{t}}\right)=\left[\begin{array}{cc}
0 & 1 \\
-\frac{1}{p_{1} p_{2}} & -\frac{p_{1}+p_{2}}{p_{1} p_{2}}
\end{array}\right]\left(\begin{array}{c}
V_{t} \\
\dot{V}_{t}
\end{array}\right)+\left(\begin{array}{c}
0 \\
\frac{k}{p_{1} p_{2}}
\end{array}\right) v
$$

where $v \in N(1,0)$ and the parameters $p_{1}, p_{2}$ and $k$ depend on the mean value of the wind speed $\left(V_{\mathrm{m}}\right)$, which is described as

$V_{\mathrm{m}}=\frac{1}{T} \int_{t-\frac{T}{2}}^{t+\frac{T}{2}} v(\tau) \mathrm{d} \tau$

\section{Nonlinear model of wind turbine system}

The wind turbine that is considered in this paper has the following specifications (Table 1).

A wind turbine consists of a set of interconnected subsystems. These subsystems include the following: aerodynamic, mechanical, generator, and driving dynamics to turn the pitch angle. Several degrees of freedom could be considered to model the structure, but for control design mostly just a few important degrees of freedom are considered. In this work we only consider two degrees of freedom, namely the rotational degree of freedom (DOF) 
Table 1 Characteristic of vestas V29 wind turbine

\begin{tabular}{ll}
\hline Vestas V29 wind turbine characteristic & Value \\
\hline Electrical power of generator & $225 \mathrm{~kW}$ \\
Rotor diameter & $29 \mathrm{~m}$ \\
Rotor RPM & $41 / 30.8 \mathrm{RPM}$ \\
Angular speed of rotor & $4.29 \mathrm{rad} / \mathrm{s}$ \\
Angular speed of generator & $105.6 \mathrm{rad} / \mathrm{s}$ \\
Frequency & $50-60 \mathrm{~Hz}$ \\
\hline
\end{tabular}

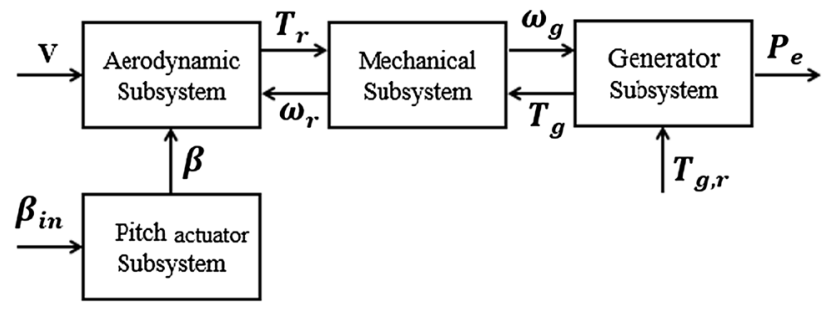

Fig. 1 Block diagram of the wind turbine

and drivetrain torsion. Figure 1 shows the interconnection between these subsystems [10].

\section{Aerodynamics subsystem}

The aerodynamics subsystem can be modeled by the following nonlinear equation [17]:

$P_{\mathrm{r}}=\frac{1}{2} \rho \pi R^{2} V^{3} C_{\mathrm{p}}(\lambda, \beta)$,

where $P_{\mathrm{r}}$ is the electrical power of rotor, $\rho$ is the density of wind $\left(\frac{\mathrm{kg}}{\mathrm{m}^{3}}\right), R$ is the radius of the blades $(\mathrm{m}), V$ is the wind speed $(\mathrm{m} / \mathrm{s}), \lambda$ is the tip speed ratio, $\beta$ is the pitch angle, $C_{\mathrm{p}}$ : the power coefficient, $\lambda$ is the ratio between the blade tip speed and the wind speed,

and $\lambda=\frac{\mathrm{V}}{R \omega_{\mathrm{r}}}$,

where the $\omega_{\mathrm{r}}$ is the angular speed of rotor. The derivative of $C_{\mathrm{p}}$ with respect to both $\lambda$ and $\beta$ is, therefore, used extensively in the control designs. In previous work, numerical derivatives of $C_{p}$ with respect to $\lambda$ and $\theta$ have been successfully obtained and utilize in linear control designs. However, the nonlinear controllers have proven sensitive towards the noise introduced by the numerical derivations, making it difficult to validate the responses obtained by, e.g. a feedback linearizing controller. Consequently, it has been chosen to base the aerodynamic model on an analytic expression of $C_{\mathrm{p}}$. The relation between $C_{\mathrm{p}}, \beta$ and $\lambda$ can be written as [17]:
$C_{\mathrm{p}}(\lambda, \beta)=0.22\left(\frac{116}{\lambda_{\mathrm{t}}}-0.6 \beta-5\right) \exp \left(\frac{12.5}{\lambda_{\mathrm{t}}}\right)$

where

$\frac{1}{\lambda_{\mathrm{t}}}=\frac{1}{\lambda^{-1}+0.12 \beta}-\frac{0.035}{(1.5 \beta)^{3}+1}$

\section{Generator subsystem}

It has been assumed that the asynchronous generator is ideal, and hence the generator power is given by [18]:

$P_{\mathrm{e}}=T_{\mathrm{g}} \omega_{\mathrm{g}}$

As mentioned, the $P_{\mathrm{e}}$ is electrical power of generator, the $\omega_{\mathrm{g}}$ is the angular speed of generator, and the $T_{\mathrm{g}}$ is the generator torque that can be controlled; however, it cannot be changed instantaneously. The dynamic response of the generator has, therefore, been modeled by a first-order linear model [10]:

$\dot{T}_{\mathrm{g}}=-\frac{1}{\tau_{\mathrm{T}}} T_{\mathrm{g}}+\frac{1}{\tau_{\mathrm{T}}} T_{\mathrm{g}, \mathrm{r}}$,

where $T_{\mathrm{g}, \mathrm{r}}$ is the reference value for the generator torque, and $\tau_{\mathrm{T}}$ is the time constant.

\section{Pitch actuator subsystem}

In order to force the blade angle $\beta$ tracks the reference blade angle $\beta_{\text {in }}$, The pitch of the blades is changed by a hydraulic/mechanical actuator. A simplified model of the dynamics is presented by the following first-order linear model [10]:

$\dot{\beta}=-\frac{1}{\tau_{\beta}} \beta+\frac{1}{\tau_{\beta}} \beta_{\text {in }}$,

where $\tau_{\beta}$ is the time constant.

\section{Mechanical subsystem}

The turbine is split into two parts, separated by the transmission: The rotor side and the generator side [19]. The inertia on the rotor side $J_{\mathrm{r}}$ and generator side $J_{\mathrm{g}}$ are illustrated by the leftmost and the rightmost disc, respectively. The shaft connecting the rotor to the transmission is subject to immense torques that cause it to twist; consequently the shaft is appropriately modeled as a damped spring. On the left the model is exited by the rotor torque $T_{\mathrm{r}}$ and on the right the generator torque $T_{\mathrm{g}}$. The torques $T_{\mathrm{sr}}$ and $T_{\mathrm{sg}}$ are the torques at each side of the transmission part and has relation by $N_{\mathrm{g}}$ as the gear ratio. 
$T_{\mathrm{sg}}=\frac{T_{\mathrm{sr}}}{N_{\mathrm{g}}}$

The equations describing the dynamics are obtained using Newton's second law for rotating bodies. This results in two equations: one for the rotor side and one for the generator side.

$\dot{\omega}_{\mathrm{r}}=T_{\mathrm{r}}-T_{\mathrm{sr}}$

$\dot{\omega}_{\mathrm{g}}=T_{\mathrm{g}}-T_{\mathrm{sg}}$

Introducing a variable $\delta$ (rad) describing the twist of the shaft leads to the following equation describing the twist of the flexible shaft [20]:

$T_{\mathrm{sr}}=D_{\mathrm{r}} \dot{\delta}+K_{\mathrm{r}} \delta$

where

$\delta=\Omega_{\mathrm{r}}-\frac{\Omega_{\mathrm{g}}}{N_{\mathrm{g}}}, \quad \dot{\delta}=\omega_{\mathrm{r}}-\frac{\omega_{\mathrm{g}}}{N_{\mathrm{g}}}$

In the above equations, $D_{\mathrm{r}}$ is damping and $K_{\mathrm{r}}$ is spring coefficient, $\omega_{\mathrm{r}}$ is angular speed of rotor, $\omega_{\mathrm{g}}$ is angular speed of generator, $\Omega_{\mathrm{r}}$ and $\Omega_{\mathrm{g}}$ are naturally the shaft angle at the rotor and the generator, respectively.

\section{Linearized models of wind turbine system}

As it was mentioned, for control design we need to have a linear model of the system. In this model, wind is considered as an input [21]:

Input $=\left[V \beta_{\text {in }} T_{\mathrm{g}, \mathrm{r}}\right]^{\mathrm{T}}$

And outputs of the system include

Output $=\left[\omega_{\mathrm{r}} \omega_{\mathrm{g}} P_{\mathrm{e}}\right]$

Having all the equations, system equations become [12]:

$\dot{\omega}_{\mathrm{r}}=\frac{a-D_{\mathrm{r}}}{J_{\mathrm{r}}} \omega_{\mathrm{r}}-\frac{D_{\mathrm{r}}}{J_{\mathrm{r}} N_{\mathrm{g}}} \omega_{\mathrm{g}}-\frac{K_{\mathrm{r}}}{J_{\mathrm{r}}} \delta+a_{14} \theta$

$\dot{\omega}_{\mathrm{g}}=\frac{D_{\mathrm{r}}}{N_{\mathrm{g}} J_{\mathrm{g}}} \omega_{\mathrm{r}}-\frac{D_{\mathrm{r}}}{N_{\mathrm{g}}^{2} J_{\mathrm{g}}} \omega_{\mathrm{g}}+\frac{K_{\mathrm{r}}}{N_{\mathrm{g}} J_{\mathrm{g}}} \delta-\frac{1}{J_{\mathrm{g}}} T_{\mathrm{g}}$

$\dot{\delta}=\omega_{\mathrm{r}}-\frac{\omega_{\mathrm{g}}}{N_{\mathrm{g}}}$

$\dot{\beta}=-\frac{1}{\tau_{\beta}} \beta+\frac{1}{\tau_{\beta}} \beta_{\text {in }}$

$\dot{T}_{\mathrm{g}}=-\frac{1}{\tau_{\mathrm{T}}} T_{\mathrm{g}}+\frac{1}{\tau_{\mathrm{T}}} T_{\mathrm{g}, \mathrm{r}}$

$\dot{V}_{\mathrm{t}}=V_{\mathrm{t}}$

$\ddot{V}_{\mathrm{t}}=-\frac{1}{p_{1} p_{2}} V_{\mathrm{t}}-\frac{p_{1}+p_{2}}{p_{1} p_{2}} \dot{V}_{\mathrm{t}}+\frac{k}{p_{1} p_{2}} v$ in which $J_{\mathrm{r}}$ and $J_{\mathrm{g}}$ are rotor and generator moments of inertia, $\tau_{\beta}$ and $\tau_{\mathrm{T}}$ are time constants of the first-order actuator models.

\section{The impact of cold weather on the operation of wind turbine}

Various uncertainties have been examined in the current literature. These uncertainties derive from approximated and process parameters in a nonlinear system which changes as the operating point changes, a matter causing the electrical power production to reduce. However, all previous control designs work at moderate temperature. Temperature reduction in cold seasons has devastating effects on the wind turbine. The existence of ice on the components of wind turbine causes some serious problems. The least amount of ice on the blades deteriorates the aerodynamic performance of the system. It not only reduces the output power, but also increases the abrasion between the components $[15,20]$. In fact, the existence of ice in cold places and the high density of air at low temperatures have detrimental effects on aerodynamics. Variation of produced power and load serve as proofs for such dysfunctionality. Ice masses on the turbine change the natural frequency of the turbine's components and also the behavior of the system's dynamic [14-16]. The control system has also been affected by these conditions. Indeed, the performance of the turbine system deteriorates through false information sending [13]. Previous papers dealing with this problem have proposed methods such as observation, the use of sensors and monitors, considering aerodynamic sound, etc. to identify ice. The control systems are then designed to destroy the ice [13, 14, 21].

In this paper, a new strategy is advanced for the first time to improve the wind turbine performance in cold weather conditions and prevent the damage which brings about the shutdown of the turbine. Due to the structure of the wind turbine, as the mass of the frozen blades changes, the rotor mass changes leading to a change in the rotor inertia. These changes will affect the equations of the wind turbine and optimal power production. Thus, turbine control and optimal power output would be possible by considering the rotor inertia as a new element of uncertainty in the system. The amount of this uncertainty varies with temperature reduction and the turbine's production capacity.

Based on the following Ref. [22], the blade imbalance was simulated by scaling the mass density of one blade, which creates an uneven distribution of mass with respect to the rotor. In addition, the aerodynamic asymmetry was 
simulated by adjusting the pitch of one blade, which creates an uneven torque across the rotor. In our paper, the blade imbalance is due to icing on the blade. Therefore, the imbalance of the blade is considered around $20 \%$ as uncertainty. We have designed a robust control to control the turbine in the presence of uncertainty due to the blade imbalance icing.

The uncertainties in the wind turbine include the linearized model parameters extracted from the nonlinear model, spring constant, and damping coefficient which change as the operating point deviates; another uncertainty is added to the system due to the existence of noise and disturbance in the input signal. All of these uncertainties are considered in suitable weather conditions. In this paper, cold weather and rotor inertia are viewed as other sources of uncertainty in the wind turbine system [23, 24]. If we place the wind turbine in Manjil, in the north of Iran, the turbine blades will freeze at the temperature below -10.3 , the lowest measured temperature in the region between years 1989 and 2013. These icy turbine blades change the rotor mass. The open loop frequency response analysis of the linearized wind turbine and all five uncertainties will be applied to determine the exact range of changes in the rotor mass. Under the frequency response analysis of the system, the rotor inertia uncertainty can be considered between the range of 0 and $20 \%$. The uncertainties in parameters can be shown as follows:

$K_{\mathrm{r}}=\bar{K}_{\mathrm{r}}\left(1+p_{K_{\mathrm{r}}} \delta_{K_{\mathrm{r}}}\right)$

$D_{\mathrm{r}}=\bar{D}_{\mathrm{r}}\left(1+p_{D_{\mathrm{r}}} \delta_{D_{\mathrm{r}}}\right)$

$a=\bar{a}\left(1+p_{a} \delta_{a}\right)$

$a_{14}=\bar{a}_{14}\left(1+p_{a_{14}} \delta_{a_{14}}\right)$

$J_{\mathrm{r}}=\bar{J}_{\mathrm{r}}\left(1+p_{J_{\mathrm{r}}} \delta_{J_{\mathrm{r}}}\right)$

There are two reasons why spring coefficient and the damping are considered as uncertainties:

1. There is deviation in the spring parameters from production to production and manufacturer to manufacturer.

2. It should be noted that the spring coefficient and damping may change in a long time due to continuous operation and aging.

In the above equations, $\bar{K}_{\mathrm{r}}, \bar{D}_{\mathrm{r}}, \bar{a}, \bar{a}_{14}$ are nominal parameter values, resulting from the spring constant, damping coefficient, linearization process $* * *$ and rotor inertia, respectively. $p_{K_{\mathrm{r}}}, p_{D_{\mathrm{r}}}, p_{a}, p_{a_{14}}$ indicate maximum relative uncertainties that are for uncertainty parameters which are shown in Table 2. $\delta_{K_{\mathrm{r}}}, \delta_{D_{\mathrm{r}}}, \delta_{a}, \delta_{a_{14}}, \delta_{J_{\mathrm{r}}}$ are relative changes in these parameters. Therefore,

$\left|\delta_{K_{\mathrm{r}}}\right| \leq 1,\left|\delta_{D_{\mathrm{r}}}\right| \leq 1,\left|\delta_{a}\right| \leq 1, \quad\left|\delta_{a_{14}}\right| \leq 1,\left|\delta_{J_{\mathrm{r}}}\right| \leq 1$
Table 2 Parameter uncertainties of the wind turbine

\begin{tabular}{llll}
\hline Parameter & Description & Unit & Tolerance \\
\hline$K_{\mathrm{r}}$ & Spring coefficient & $\mathrm{Nm} / \mathrm{rad}$ & $\pm 10 \%$ \\
$D_{\mathrm{r}}$ & Damping & $\left(\frac{\mathrm{kg} \mathrm{m}^{2}}{\mathrm{rads}}\right)$ & $\pm 10 \%$ \\
$A$ & Linearization parameter & - & $\pm 20 \%$ \\
$a_{14}$ & Linearization parameter & - & $\pm 20 \%$ \\
$J_{\mathrm{r}}$ & Rotor inertia & $\mathrm{kg} \mathrm{m}^{2}$ & $+20 \%$ \\
\hline
\end{tabular}

\section{Robust control design}

\section{Closed-loop system design specifications}

Figure 2 shows the block diagram of wind turbine closedloop system, including the feedback structure, the controller, as well as the model uncertainties and performance objectives weights.

In the above figure, $(r)$ is the reference input, $(V)$ is the wind speed with disturbance, $(n)$ is noise, and $e_{\mathrm{u}}$ and $e_{\mathrm{y}}$ are two output costs. The system $(R)$ is an ideal model of performance, to which the designed closed-loop system tries to match [25]. The model transfer function is chosen so that the time response to the reference signal would have an overshoot less than 5\%. Inside the rectangle is the nominal model $G_{\text {nom }}$ of the wind turbine plant and the block $\Delta$ that parameterises the model uncertainties. To obtain the desired performance, we need to find the transfer function matrix from inputs $r$, and disturbance in $V$ and $n$ to outputs $e_{\mathrm{u}}$ and $e_{\mathrm{y}}$ so that the infinity norm of that transfers function is small for all available uncertainty parameter values. The position noise signal is obtained by passing the unit-bounded signal $n$ through the weighting transfer matrix $W_{\mathrm{n}}$. The transfer matrices $W_{\mathrm{p}}$ and $W_{\mathrm{u}}$ are used to reflect the relative significance of the different frequency ranges for which the performance is required. Hence, the performance objective can be recast, with possible slight conservativeness, as that the infinity norm of the transfer function matrix is less than 1 . Then $\Delta$ matrix is defined as follows:

$\Delta=\operatorname{diag}\left(p_{K_{\mathrm{r}}}, p_{D_{\mathrm{r}}}, p_{a}, p_{a_{14}}, p_{J_{\mathrm{r}}}\right)$

This transfer function can be written as [25]:

$$
\left[\begin{array}{c}
e_{\mathrm{y}} \\
e_{\mathrm{u}}
\end{array}\right]=\left[\begin{array}{ccc}
W_{\mathrm{p}}\left(S_{\mathrm{o}} G_{\mathrm{u}} K-R\right) & W_{\mathrm{p}} S_{\mathrm{o}} G_{\mathrm{v}} & -W_{\mathrm{p}} S_{\mathrm{o}} G_{\mathrm{u}} K W_{\mathrm{n}} \\
W_{\mathrm{u}} S_{\mathrm{i}} K & -W_{\mathrm{u}} K S_{\mathrm{o}} G_{\mathrm{v}} & -W_{\mathrm{u}} K S_{\mathrm{o}} W_{\mathrm{n}}
\end{array}\right]\left[\begin{array}{c}
r \\
V \\
n
\end{array}\right],
$$

where $S_{\mathrm{i}}=(I+K G)^{-1}$ and $S_{\mathrm{o}}=(I+G K)^{-1}$ are the input and output sensitivities, respectively. Note that $S_{\mathrm{o}} G$ is the transfer function between $V$ and $y$. 
Fig. 2 Block diagram of the closed-loop system with performance specifications

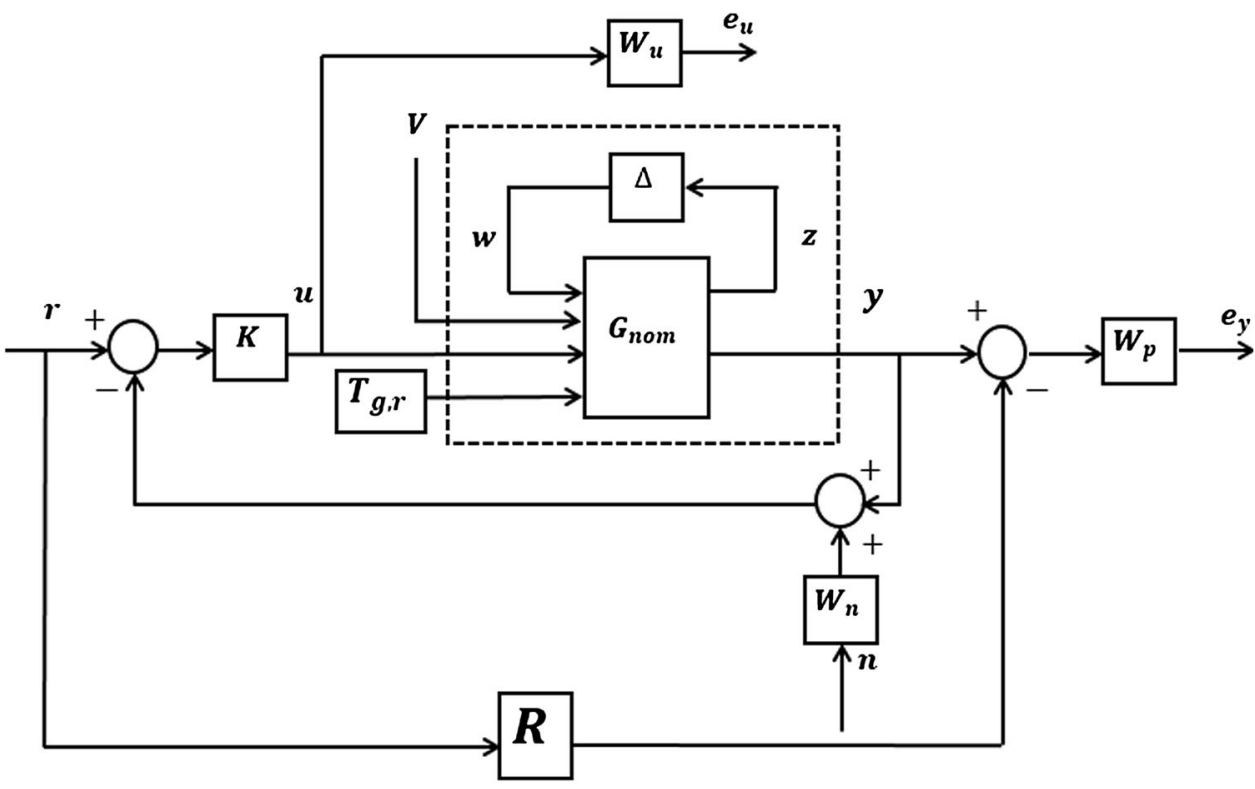

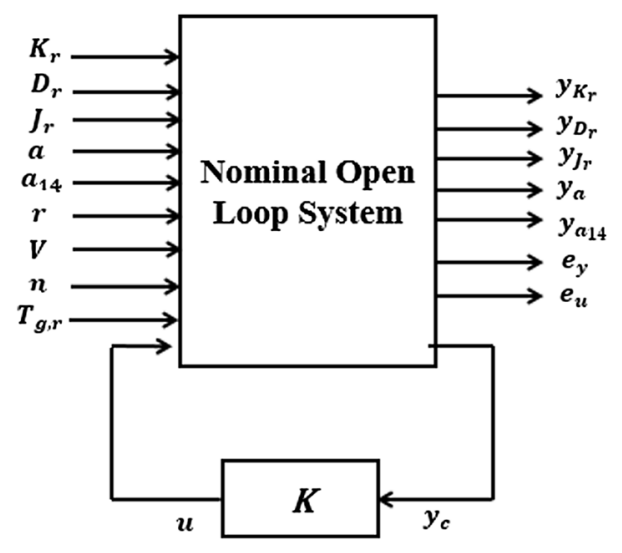

Fig. 3 Closed-loop system model for design $\mu$ robust controller

\section{Robust $\mu$ controller design}

The aim of control by using the $\mu$ synthesis method was the stability of the closed-loop system and satisfying all of the control requirements. The closed-loop system must have the robust performance in the presence of uncertainties, disturbance in the wind, and measurement noise. As a result, it can be said that the aim of $\mu$ controller design is that the wind turbine can follow the reference input of produced electrical power in the presence of disturbance and noise. In Fig. 3, the block diagram of the closed-loop system that is used with the defined uncertainties is demonstrated for $\mu$ controller system design.

From the figure, it can be seen that $y_{\mathrm{c}}$ is defined as the difference between noisy output and the reference and the transfer function $P(s)$ shows the open-loop transfer function matrix with ten inputs and eight outputs. The upper linear fractional transformation (LFT) of the closed-loop system is

$P=F_{\mathrm{u}}\left(P_{\text {nom }}, \Delta_{\mathrm{r}}\right)$,

where $P_{\text {nom }}$ is the nominal transfer function matrix, $\Delta_{\mathrm{r}}$ includes five uncertainties in the wind turbine model. We assume $\Delta_{\mathrm{P}}$ is defined the structure of uncertainties block as follows:

$\Delta_{\mathrm{P}}:=\left\{\left[\begin{array}{cc}\Delta_{\mathrm{r}} & 0 \\ 0 & \Delta_{\mathrm{F}}\end{array}\right]: \Delta_{\mathrm{r}} \in \mathcal{R}^{5 \times 5}, \Delta_{\mathrm{F}} \in C^{4 \times 2}\right\}$

\section{Robust stability}

By definition, the closed-loop system achieves robust stability if the closed-loop system is internally stable for each possible plant dynamics $G=F_{\mathrm{u}}\left(G_{\mathrm{nom}}, \Delta\right)$.

\section{Robust performance}

The closed-loop system must remain internally stable for each $G=F_{\mathrm{u}}\left(G_{\text {nom }}, \Delta\right)$ and in addition the performance criterion should be satisfied for each $G=F_{\mathrm{u}}\left(G_{\mathrm{nom}}, \Delta\right)$ [25]. Controller that is obtained with this method is usually of high order, which leads to problems in a practical implementation. So often, it is useful to reduce the order of the control system as much as possible to simplify the closedloop system analysis and implementation. The results of the structured singular value is calculated after repeating 5 D-K steps. These results are in the Table 3.

It can be seen that the maximum value of $\mu$ is 11.648 that is achieved in the first iteration. Similar to this method, next steps are continued in order to $\gamma$ be less than 1 . The designed $\mu$ controller synthesis is of order 17 , and it is 
Table 3 Summary of results of $\mu$ robust control

\begin{tabular}{lccc}
\hline Iteration number & $\gamma$ value achieved & Maximum $\mu$ value & Controller order \\
\hline 1 & 1201.403 & 11.648 & 5 \\
2 & 5.35 & 3.837 & 11 \\
3 & 1.763 & 0.748 & 16 \\
4 & 0.734 & 0.733 & 17 \\
5 & 0.732 & 0.730 & 17 \\
\hline
\end{tabular}

achieved after five iterations. In the final iteration, the value of $\gamma$ reached 0.732 and, $\mu$ reaches 0.730 which is less than 1 . This means that the closed-loop system has robust performance, because the structured singular value is less than 1 in any frequency. The gamma value also represents the value that the infinity norm of function $F_{1}(P, K)$ was less than that value.

\section{$\mu$ Controller order reduction}

By using $\mu$ synthesis, both for low-order and high-order systems, the resultant controller will be of high order. This causes problems such as increased calculations and complexity of the implementation. In this paper, for the first time, Henkel norm approximation method is used [25, 26]. In Henkel norm approximation, first the minimality of the system should be checked, and then it eliminates uncontrollable and unobservable modes. In the following, Henkel singular values are obtained and low-energy singular values are neglected to obtain the reduced order controller. Full order $\mu$ controller is of order 17. By reducing order of control system, hardware implementation will be easier. Considering tradeoff between the control specifications and order of the control system, we can reduce $\mu$ controller to 11.

\section{Simulation results of the designed robust controller and PID controller comparison}

\section{PID controller design}

In this section, by considering the uncertainties in wind turbine system model, the PID controller is designed to control electrical power and adjust the speed of generator. Its control performance is finally compared with robust controller performance that is designed in previous section.

The standard formula of this system is as follows [27]:

$K_{\mathrm{p}}\left(1+\frac{1}{T_{\mathrm{i}} s}+T_{\mathrm{d}} s\right)$

where $K_{\mathrm{p}}, T_{\mathrm{i}}$ and $T_{\mathrm{d}}$ are the proportional gain, the integral and derivative time constants, respectively.

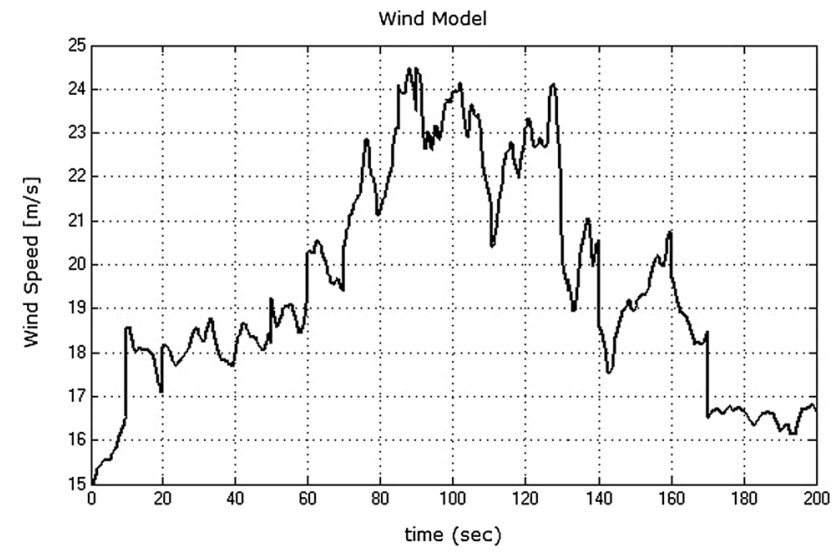

Fig. 4 Wind model in different speeds

\section{Simulation results}

Considered wind model in this paper is as follows.

Figure 4 exhibits the wind speed in the third area which is between 15 and $25 \mathrm{~m} / \mathrm{s}$. In this paper, the wind model has randomly changed from 15 to $24 \mathrm{~m} / \mathrm{s}$ (another suggestion: In this paper, the wind model varies from 15 to $24 \mathrm{~m} / \mathrm{s}$ ). In our paper, the wind speed is considered be highly variable at different times $[10,11]$. As in early times the wind turbine is increasing, then its value is fixed in time and its value dropped in the end times. The reason for choosing this type of model of the random wind is showing the robust performance of controller in different speeds.

This means that a sudden increase or decrease in wind speed, the robust controller tries to control the pitch angle for fixing the electrical power at its maximum performance in the third performance section.

Because of the linear dependency between the rotor speed and the generator speed through the gear ratio, the results of applying the control method-which seeks to keep the electrical power constant and adjust generator speed-will be examined after modeling of the wind turbine and designing the robust $\mu$ controller.

Considering Fig. 5a, the deviation of output around the nominal value that is affected by noise and disturbance in wind is very little. Maximum changes around the reference value are $2.1 \%$ and it is equivalent to $0.027 \mathrm{~kW}$ which represents good disturbance rejection in areas in the matching model. In Fig. 5b, maximum changes around 
nominal values are equal to $3.068 \mathrm{rad} / \mathrm{s}$ and it is equivalent to $9.2 \%$. It represents a disturbance rejection and good tracking of generator reference value in the presence of disturbance with wind speed in the best way and with the least changes.

In Fig. 6, it can be noted that the control effort or the adjustment of pitch angle, is in the range of $14^{\circ}-22.5^{\circ}$. Because of different areas of wind turbine performance, purpose in this article is, fixing the power and speed of the generator at the nominal value in the third performance section. Therefore, in this area by increasing the amount of wind to cut-out, the pitch angle has been increased, and this condition causes the reduction of power coefficient and the power is at its nominal value [4].

In addition, by decreasing the wind speed, the blade pitch angle is reduced and at this step to fix the power and speed of the generator at nominal value, robust controller was designed. This controller is tried to control the pitch angle for accessing the maximum power electric and tune

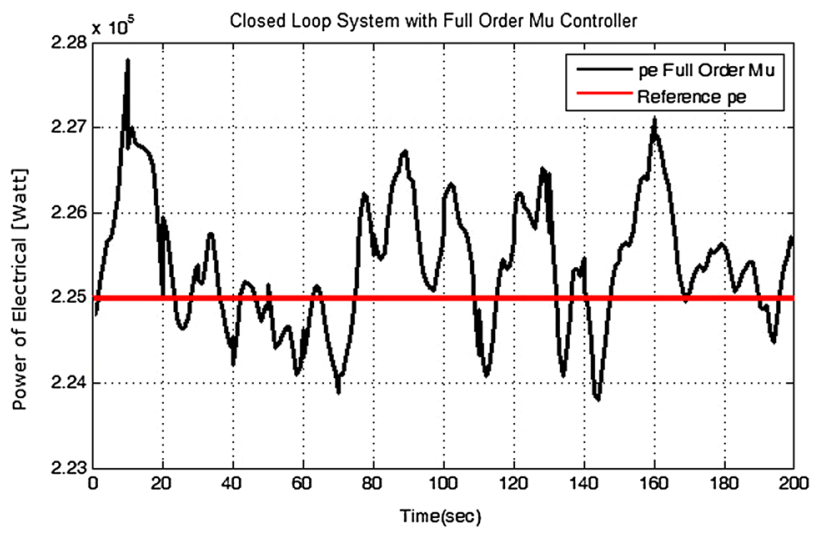

(a)

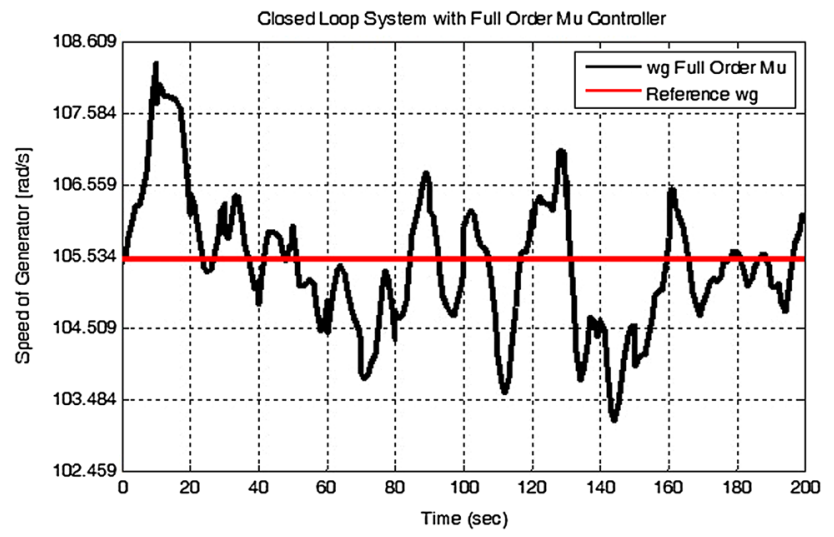

(b)

Fig. 5 a The response of electric power produce by wind turbines to track input reference electrical power using $\mu$ controller. b The response of speed of the wind turbine generator to track input reference speed of generator using $\mu$ controller the speed of generator around its nominal value. As a result, by utilizing this type of controller, control effort remains less than $25^{\circ}$, which is the maximum pitch angle of the wind turbine, remains limited in the third area.

In this section, results of the wind turbine using reduced order $\mu$ controller is studied.

As it can be seen in Fig. 7, the comparison the output electric power of full order and reduced order controllers. It is concluded that the most changes around nominal value in the reduced order control system is equal to $0.89 \%$ which is equivalent to $0.02 \mathrm{~kW}$, compare to full order controller changes decreased to the $0.31 \%$.

From Fig. 8, the maximum amount of the changes around of nominal value is increased by using of reduced order controller and it is equal to $3 \%$ which is equivalent to $3.074 \mathrm{rad} / \mathrm{s}$ that is increased to $0.006 \mathrm{rad} / \mathrm{s}$. This increase shows the poor performance of reduced

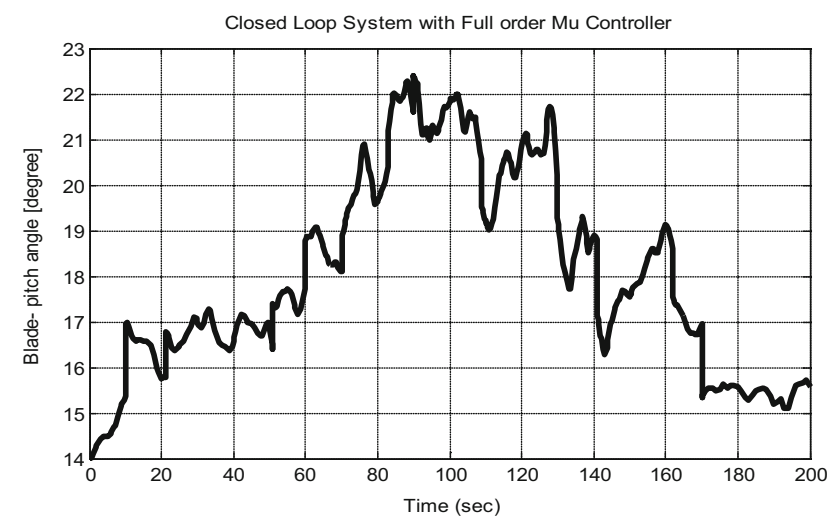

Fig. 6 Pitch angle adjustment using the $\mu$ controller

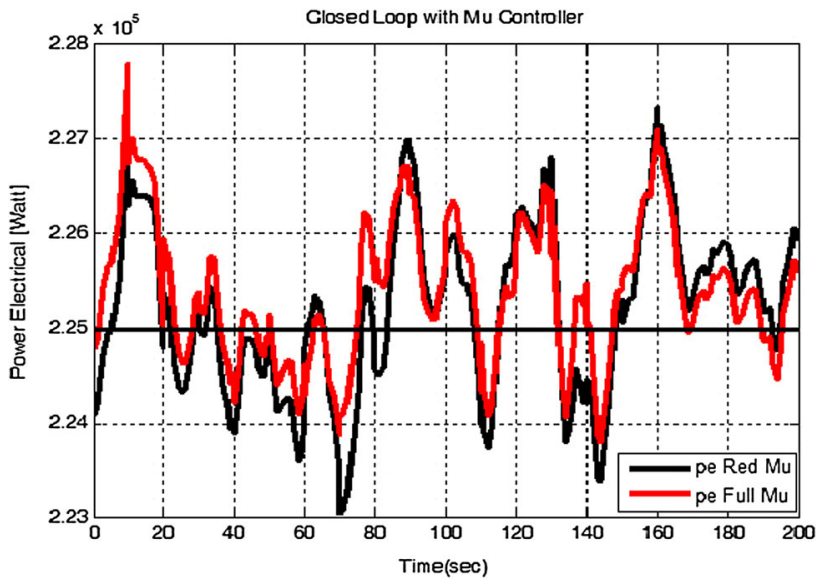

Fig. 7 Comparison of response of produced electrical power to reference electrical power using reduced order and full-order $\mu$ controllers 


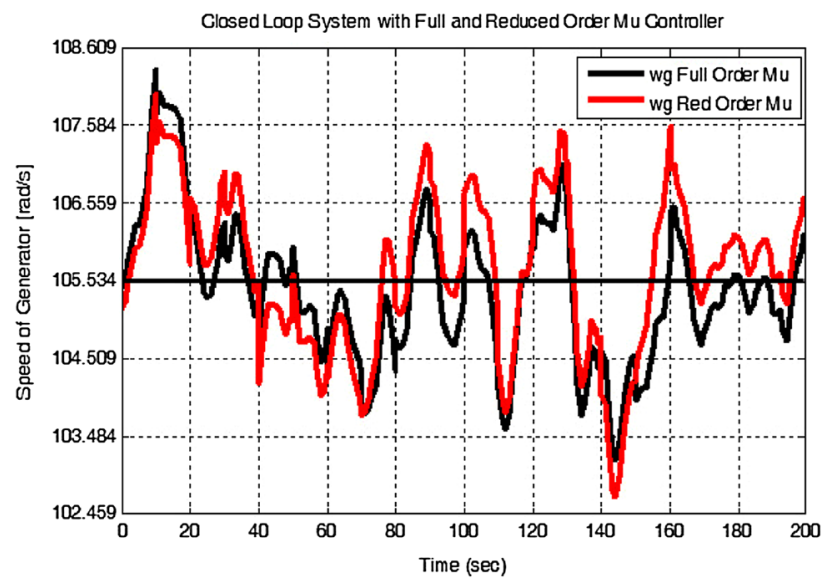

Fig. 8 Comparison between response of speed generator to reference speed generator using reduced order and full-order $\mu$ controllers

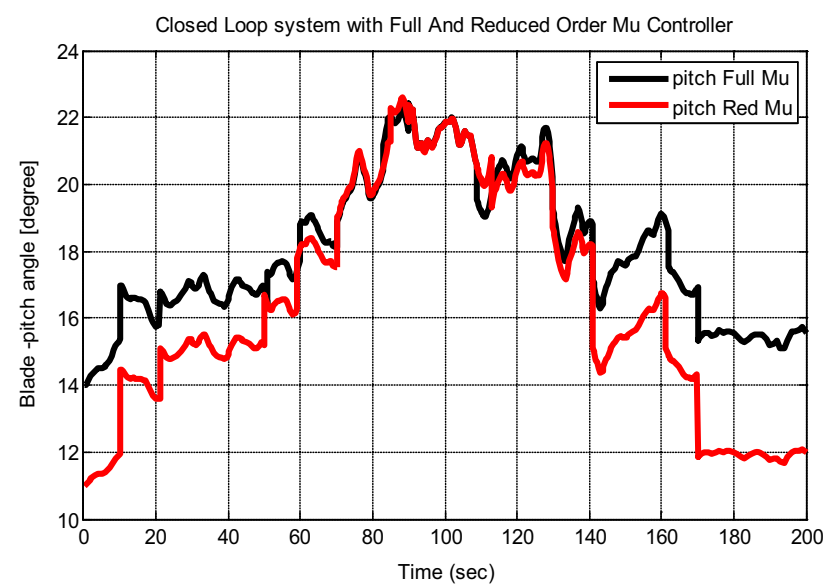

Fig. 9 Comparison between adjustments of pitch using reduced order and full-order $\mu$ controllers

order controller in rejecting disturbance and also to weaken the noise effect compared to full order controller. However, the control system has been increased the changes compared its full order controller, but reduces it compared to uncontrolled system, and it is located within the limits of matching model and used for simplicity in implementation.

Utilizing reduced order robust control system in Fig. 9, control effort which is in degrees and should be in the range of $0^{\circ}$ and $25^{\circ}$, has a value between $11^{\circ}$ and $22.5^{\circ}$. In order to achieve the optimal electrical power and reduction of variation around the nominal value, more efforts should be applied to control the blade angle. It represents an increase of variations, control efforts, and lower performance of reduced order controller compared to full order controller. Thus, one can say that reduced order controller is only suitable for simplicity in the implementation and a

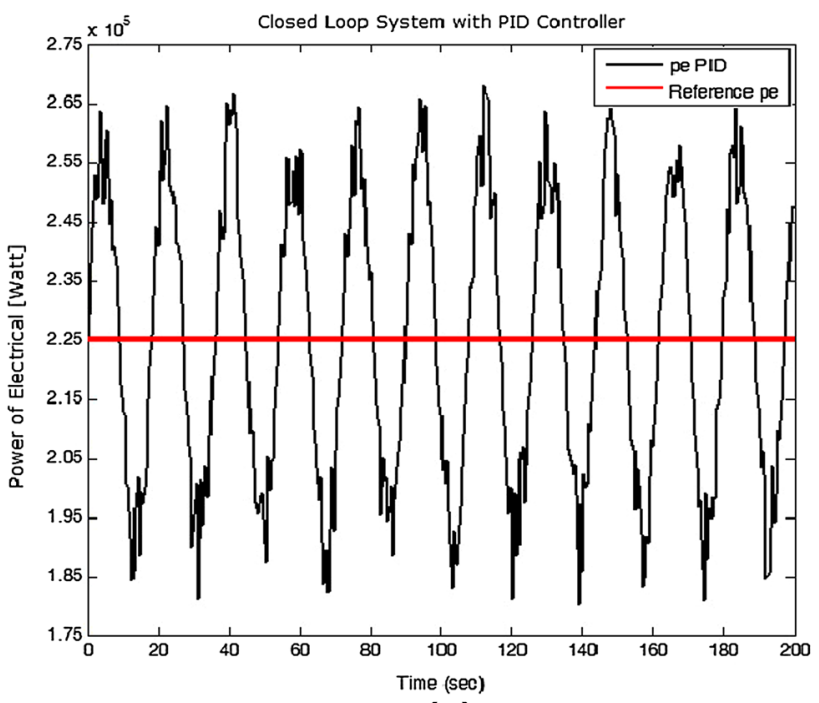

(a)

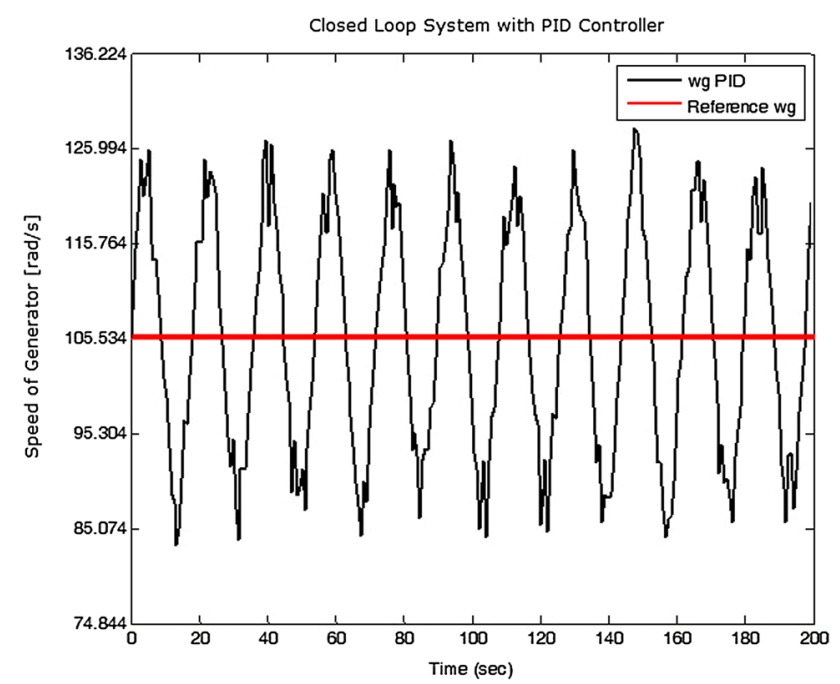

(b)

Fig. 10 a The response of electric power produced by wind turbines to track input reference electrical power by using the PID controller. b The response of speed of the wind turbine generator to track input reference speed of generator by using the PID controller

tradeoff should be considered. In the following, the simulation results are shown using the designed PID controller.

According to the results in Fig. 10, it can be seen that using PID controller in wind turbine system is not appropriate by considering uncertainties. Variations around nominal values for output power is equal to $17 \%$ in Fig. 10a and the output generator speed is 19\% in Fig. 10b. According to the control criteria of the matching model, the $5 \%$ error rate is considered. It represents a poor performance of the controller in the rejection of disturbance and noise. The dominant frequency based on the frequency period is $0.05 \mathrm{~Hz}$, and it is shown that its performance is same to low pass filter. 


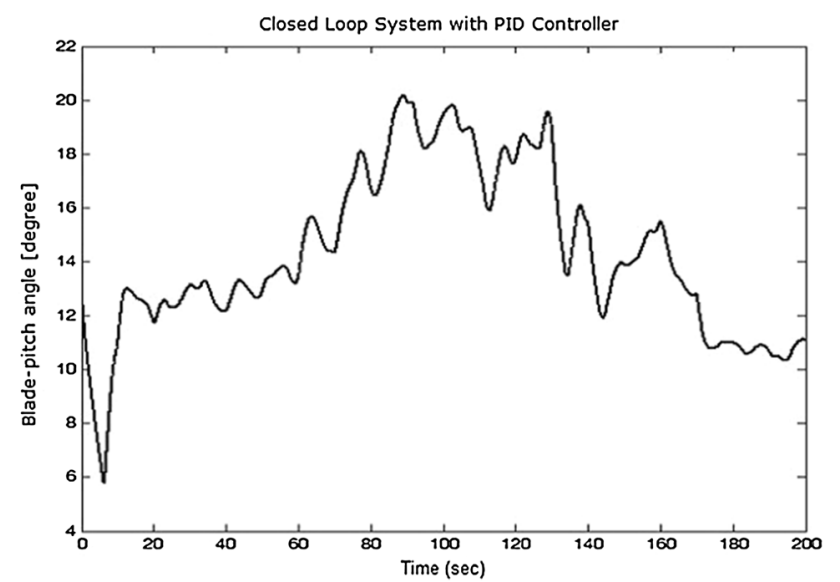

Fig. 11 Pitch angle adjustment using the PID controller

Pitch angle changes at different wind speed in presence of PID controller is shown in Fig. 11.

In above figure, the changing of pitch angle in PID controller is among $6-19^{\circ}$ that it is shown the input controller for pitch angle is high. In addition, it is shown in first by increasing the speed wind, the pitch angle is reduced suddenly. We can include that in first time the PID controller could not control the pith angle for use of maximum power electric when wind speed is increased.

According to changing range of pitch angle in full order $\mu$, it is consulted that the input effort of PID controller is more than in full order $\mu$. Therefore, the controller is weaker than full order $\mu$.

Now, we compare the robust controllers with PID controllers that are presented in this paper. To analyze the performance of controllers, mean square error (MSE) is considered as a criterion. The results of this evaluation in electric power control and adjustment of the speed of the generator are shown in Table 4.

According to above tables, it can be seen that by applying robust $\mu$ control, the mean squared error has a minimum value compared to PID controller and reduced order $\mu$.

The most mean square error value is belongs to the PID controller that it is represents a good rejection of disturbance and weak the noise compared with reduced order $\mu$.
By considering the above table, it can be said that the best option is $\mu$ control system, and then reduced order $\mu$ is the best. Also it is not suggested to use PID controllers, because it has poor performance in disturbance rejection and noise attenuation.

\section{Conclusion}

This paper attempted to control the pitch angle of the wind turbine in order to adjust the speed of the wind turbine generator in the third area of operation. In the third area, the speed of generator, and electrical power are constant in their nominal values, and do not change.

The presence of noise and disturbance in the wind model are the main causes for high error rate in the production of electrical power and generators' speed. Thus, this article proposed that the production of electrical power and adjustment of the generator speed are feasible if robust controller is applied and current errors (uncertainties) in the wind turbine system are taken into account. In most reference works, spring constant, damping coefficient and insignificant deviations of the linearization process are listed as uncertainties. These uncertainties are deemed to be true in suitable weather conditions. However, lower temperature causes the turbine blades to freeze which is followed by mass increase. This mass increase results in the reduction of electrical power production, incorrect system operation and false information sending. Therefore, new uncertainties were applied to the system in order to solve the mentioned problems. After applying these uncertainties, full and reduced-order $\mu$ controllers were designed. This was followed by comparing the results of electrical power and the generator's speed with PID controller through the mean square error criterion. Simulation results show that the PID controller is not appropriate for the wind turbine system in the presence of uncertainties and has the greatest changes around the nominal value indicating the poor performance of the controller in rejecting disturbance. Therefore, minimal changes around the reference value and less control effort for changing the blades' angle (to obtain optimal power and adjust the speed of the generator) require the use of $\mu$ controller. To be more

Table 4 The mean square error obtained (changes around of nominal value) using the designed controllers to control the electric power by wind turbine and adjustment of the generator speed by wind turbine

\begin{tabular}{lcrr}
\hline Mean square error & $\mu$ controller & Reduced order $\mu$ controller & PID controller \\
\hline The control method to control the electric power & 3.24 & 3.65 & 25.31 \\
The control method for adjust the speed of the generator & 308.24 & 343.00 & 4322.53 \\
\hline
\end{tabular}


precise, the $\mu$ controller is more justifiable than the reduced order $\mu$ control system in terms of disturbance rejection.

Open Access This article is distributed under the terms of the Creative Commons Attribution 4.0 International License (http://crea tivecommons.org/licenses/by/4.0/), which permits unrestricted use, distribution, and reproduction in any medium, provided you give appropriate credit to the original author(s) and the source, provide a link to the Creative Commons license, and indicate if changes were made.

\section{References}

1. Hau, E.: Wind Turbines: Fundamental, Technologies, Application, Economics Handbook, 3rd ed (2013)

2. Blaabjerg, F., Ma, K.: Future on power electronics for wind turbine systems. IEEE J. Emerg. Select. Top. Power Electron. 1, 139-152 (2013)

3. Kaldellis, J., Zafirakis, D.: The wind energy revolution: a short review of a long history. Renew. Energy 36, 1887-1901 (2011)

4. Bazilevs, Y., Korobenko, A., Deng, X., Yan, J.: Novel structural modeling and mesh moving techniques for advanced fluidstructure interaction simulation of wind turbines. Int. J. Numer. Meth. Eng. 102, 766-783 (2015)

5. Van, T.L., Nguyen, T.H., Lee, D.C.: Advanced pitch angle control based on fuzzy logic for variable-speed wind turbine system. IEEE Trans. Energy Conv. 30, 578-587 (2015)

6. Chen, J., Chen, J., Gong, Ch.: New overall power control strategy for variable- speed fixed pitch wind turbines within the whole wind velocity range. IEEE Trans. Ind. Electron. 60, 2652-2660 (2013)

7. Kamal, E., Aitouche, A., Ghorbani, A., Bayart, M.: robust fuzzy fault-tolerant control of wind energy conversion systems subject to sensor faults. IEEE Trans. Sustain. Energy 3, 231-241 (2012)

8. Abbas, F.A., Abdulsada, M.A., Abusief, F.R.: Speed control of wind turbine by using PID controller. Int. J. Eng. Technol. 29, 65-71 (2011)

9. Evangelista, C., Valenciaga, F., Puleston, P.: Active and reactive power control for wind turbine based on a MIMI 2-sliding mode algorithm with variable gains. IEEE Trans. Energy Conv. 28, 682-689 (2013)

10. Mirzaei, M., Henrik, H., Niemannand Kjolstad, N.: A $\mu$ synthesis approach to robust control of a wind turbine. In: Proceedings of 50th IEEE Conference on Decision and Control and European Control Conference Orlando, pp. 645-650 (2011)

11. Mirzaei, M., Henrik, H., Niemannand Kjolstad, N.: DK-iteration robust control design of a wind turbine. In: IEEE International Conference on Control Applications Part of 2011 IEEE MultiConference on Systems and Control Denver, pp. 1493-1498 (2011)
12. Etemaddar, M., Hansen, M.O.L., Moan, T.: Wind turbine aerodynamic response under atmospheric icing conditions. J. Wind Energy 17, 241-265 (2014)

13. Shajiee, S., Pao, L.Y., Wagner, P.N., Moore, E.D., Mcleod, R.R: Direct ice sensing and localized closed-loop heating for active deicing of wind turbine bladed. In: Proceedings of American Control Conference Washington, pp. 634-639 (2013)

14. Sunden, B., Wu, Z.: on icing and icing Mitigation of wind turbine blades in cold climate. J. Energy Resour. Technol. 137, 1-10 (2015)

15. Jafarnejadsani, H., Pieper, J.: Gain-scheduled $l_{1}$-optimal control of variable-speed-variable-pitch wind turbines. IEEE Trans. Control Syst. Technol. 23, 372-379 (2015)

16. Iyasere, E., Salah, M., Dawson, D., Wagner, J., Tatlicioglu, E.: Optimum seeking-based non-linear controller to maximise energy capture in a variable speed wind turbine. IET Control Theory Appl. 6, 526-532 (2012)

17. Thomsen, S.C.: Nonlinear Control of Wind Turbine, Master Thesis (2007)

18. Tang, X., Deng, W., Qi, Z.: Investigation of the dynamic stability of microgrid. IEEE Trans. Power Syst. 29, 698-706 (2014)

19. Girsang, I.P., Dhupia, J.S., Muljadi, E., Singh, M., Pao, L.Y.: Gearbox and drivetrain models to study dynamic effects of modern wind turbines. IEEE Trans. Ind. Appl. 50, 3777-3786 (2014)

20. Shahriar, M.R., Borghesani, P., Tan, A.C.C.: Speed-based diagnostics of aerodynamic and mass imbalance in large wind turbines. In: Proceedings of IEEE International Conference on Advanced Intelligent Mechtronics, pp. 796-801 (2015)

21. Habibi, H., Cheng, L., Zhang, H., Kappatos, V., Selcuk, C., Gan, T.-H.: A dual de-icing system for wind turbine blades combining high- power ultrasonic guided waves and low-frequency forced vibrations. Renew. Energy 83, 859-870 (2015)

22. Gong, X., Qiao, W.: Simulation investigation of wind turbine imbalance fault. In: International Conference on Power System Technology (2010)

23. Xiu-Xing, Y., Yong-gang, L., Wei, L., Ya-jing, G., Xiao-Jun, W., Peng-fei, L.: Design, modeling and implementation of a novel pitch angle control system for wind turbine. Renew. Energy 81, 599-608 (2015)

24. Lamraoui, F., Fortin, G., Benoit, R., Perron, J., Masson, C.H.: Atmospheric icing impact on wind turbine production. Int. J. Cold Regions Sci. Technol. 100, 36-49 (2014)

25. Skogestad, S., Postlethwaite, I.: Multivariable Feedback Control Analysis and Design. Wiley, New York (2013)

26. Gu, D.W., Petkov, P.H., Konstantinov, M.M.: Robust Control Design with Matlab. Springer, Berlin (2013)

27. Safikhani, H., Tabatabaei, M.: Transient response control of first order plants based on PID controller. Majlesi J. Mechatron. Syst. 4, 17-20 (2015) 